\title{
Acute serious rebleeding after angiographically successful coil embolization of ruptured cerebral aneurysms
}

\author{
Dong-Hun Kang • Yong-Sun Kim
}

Received: 28 October 2010 /Accepted: 30 October 2010 /Published online: 16 November 2010

(C) Springer-Verlag 2010

Response to the letter to the editor

First of all, we really appreciated the comments to our published paper. In addition, we also strongly agree with the points you indicated - the definition of "successfully embolized aneurysm." At the time of designing and writing the paper, we found some evident and important patients who had re-bled even after their angiographically successfully embolized aneurysm. Therefore, we decided to write the paper, but the biggest problem then was the definition of successful embolization of the ruptured aneurysm. Since the study was retrospective, we only had information of the aneurysm size and the lists of inserted coils. So, we thought that the parameter "calculated coil density" (CCD) was the only possible manner for us to define successful embolization with objective validity. Actually, at the time of writing the paper, we had the same careful considerations after reading your paper [1] - the reliability of CCD compared with the computerized occlusion rate (COR); however, we inevitably used the tool, CCD, due to the lack of data in our coiling registry, but as you cited in the opening part of the letter to the editor, the focus of the study was not to find the most reliable factor to estimate the percentage of coil emboliza- tion, but it was the point that rebleeding potentially could occur at the acute post-embolization period in the limited cases, even in the cases of supposed successfully embolized aneurysm from our hypothesis and your hypothesis.

In our opinion, Sherif et al. also may agree to the points of our conclusion. Furthermore, the prospective study using the more precise parameter of COR is also recommended for us to re-evaluate the incidence of this kind of rebleeding. If you are available, we hope to share the data and evaluate prospectively with you the concept of acute rebleeding after successful embolization.

Conflicts of interest None.

\section{Reference}

1. Sherif C, Bavinzski G, Dorfer C, Kanz F, Schuster E, Plenk H Jr (2009) Computerized assessment of angiographic occlusion rate and coil density in embolized human cerebral aneurysms. AJNR Am J Neuroradiol 30:1046-1053
D.-H. Kang • Y.-S. Kim $(\bowtie)$

Departments of Neuroradiology, School of Medicine, Kyungpook

National University,

50, Samduk-2-ga, Jung-gu,

Daegu, Republic of Korea 700-721

e-mail:kdhdock@hotmail.com 